M.-O. Berger, R. Deriche, I. Herlin, J. Jaffré, J.-M. Morel (Eds.): ICAOS '96: Images, Wavelets and PDEs. Lecture Notes in Control and Information Sciences, Vol. 219, Springer, London, pp. 111-118, 1996.

\title{
Nonlinear Diffusion Scale-Spaces: From the Continuous to the Discrete Setting
}

\author{
Joachim Weickert \\ RWCP ${ }^{1}$ Novel Function SNN $^{2}$ Laboratory, \\ Imaging Center Utrecht, Utrecht University Hospital, \\ Heidelberglaan 100, E01.334, NL-3584 CX Utrecht. \\ Tel.: +31 30250 8377, Fax: +31302513399. \\ E-mail: Joachim.Weickert@cv.ruu.nl
}

\begin{abstract}
A survey on continuous, semidiscrete and discrete well-posedness and scale-space results for a class of nonlinear diffusion filters is presented. This class does not require any monotony assumption (comparison principle) and, thus, allows image restoration as well. The theoretical results include existence, uniqueness, continuous dependence on the initial image, maximumminimum principles, average grey level invariance, smoothing Lyapunov functionals, and convergence to a constant steady state.
\end{abstract}

Keywords. scale-space, nonlinear diffusion, discrete smoothing transformations.

\section{Introduction}

In the last years nonlinear diffusion filtering has been established as a successful tool for image smoothing and restoration. Strict scale-space results have been found recently for the continuous case [16]. The goal of the present paper is to outline how they can be extended to the semidiscrete and discrete setting. This is of significant practical importance, since a scale-space representation cannot perform better than its discrete realization.

The paper is organized as follows: Section 2 reviews well-posedness and scalespace results in the continuous framework and interprets the meaning of the obtained results. Section 3 introduces the requirements that we need in order to establish similar properties in a semidiscrete setting. Finally, Section 4 gives related results for the fully discrete framework which utilizes a finite number of scales. We conclude with a summary in Section 5 .

\footnotetext{
${ }^{1}$ Real World Computing Partnership

${ }^{2}$ Dutch Foundation for Neural Networks
} 


\section{Continuous Case}

We are concerned with a class of nonlinear diffusion processes using a diffusion tensor $D$. This symmetric positive definite matrix can be adapted to the local image structure by means of the so-called structure tensor (scatter matrix, second-moment matrix) $J_{\rho}\left(\nabla u_{\sigma}\right):=K_{\rho} *\left(\nabla u_{\sigma} \nabla u_{\sigma}^{T}\right)$, where $\nabla u_{\sigma}:=\nabla K_{\sigma} * u, K_{\sigma}$ denotes a Gaussian with standard deviation $\sigma$, and $*$ is the convolution product [17]. Matrices of this type play an important role in the local structure analysis of textures, corners and T-junctions, shape cues and spatio-temporal images $[14,3,12,8,11,2]$. The regularization parameter $\sigma>0$ makes the structure tensor insensitive to noise of order $\sigma$, while the integration scale $\rho$ gives the window size over which the orientation information is averaged. The structure tensor generalizes the edge detector $\nabla u_{\sigma}$ and provides us with additional information, for instance about corners and coherent structures. Its use for steering nonlinear diffusion filters in order to enhance coherent flow-like structures has been demonstrated in [17].

Let us consider an open rectangle $\Omega \subset \mathbb{R}^{2}$ as image domain and let a (monochromatic) image be represented by a function $f \in \mathrm{L}^{\infty}(\Omega)$. The initial boundary value problem we are concerned with is as follows ( $n$ denotes the normal vector and $\langle.,$. is the Euclidean scalar product):

Assume that $f \in \mathrm{L}^{\infty}(\Omega), \rho \geq 0$, and $\sigma, T>0$.

Let $a:=\operatorname{essinf}_{\Omega} f, b:=\operatorname{ess}_{\sup _{\Omega}} f$, and consider the problem

$$
\begin{array}{rll}
\partial_{t} u=\operatorname{div}\left(D\left(J_{\rho}\left(\nabla u_{\sigma}\right)\right) \nabla u\right) & \text { on } & \Omega \times(0, T], \\
u(x, 0)=f(x) & \text { on } & \Omega, \\
\left\langle D\left(J_{\rho}\left(\nabla u_{\sigma}\right)\right) \nabla u, n\right\rangle=0 & \text { on } & \partial \Omega \times(0, T]
\end{array}
$$

where the diffusion tensor $D=\left(d_{i j}\right)$ satisfies

(C1) Smoothness:

$$
D \in \mathrm{C}^{\infty}\left(\mathbb{R}^{2 \times 2}, \mathbb{R}^{2 \times 2}\right) .
$$

(C2) Symmetry: $d_{12}(J)=d_{21}(J)$ for all symmetric matrices $J \in \mathbb{R}^{2 \times 2}$.

(C3) Uniform positive definiteness:

For all $w \in \mathrm{L}^{\infty}\left(\Omega, \mathbb{R}^{2}\right)$ with $|w(x)| \leq K$ on $\bar{\Omega}$, there exists a positive lower bound $\nu(K)$ for the eigenvalues of $D\left(J_{\rho}(w)\right)$.

We observe that $\left(P_{c}\right)$ may comprise linear diffusion filters [9] as well as certain nonlinear models $[5,19,15,16,17]$. This class reveals the subsequent properties ([18], see also [5] for a proof of the major part of (a)):

Theorem 1 (Properties of the continuous filter class)

For the continuous filter class $\left(P_{c}\right)$ the following statements are valid:

(a) (Well-posedness and regularity results)

For any $T>0$, the problem $\left(P_{c}\right)$ has a unique solution $u(x, t)$ in the distributional sense. This solution satisfies $u \in \mathrm{C}\left([0, T] ; \mathrm{L}^{2}(\Omega)\right) \cap L^{2}\left(0, T ; H^{1}(\Omega)\right)$ 
and $\partial_{t} u \in L^{2}\left(0, T ;\left(H^{1}(\Omega)\right)^{\prime}\right)$. Moreover, $u \in \mathrm{C}^{\infty}(\bar{\Omega} \times(0, T])$, and it depends continuously on $f$ with respect to the $\mathrm{L}^{2}(\Omega)$ norm.

(b) (Extremum principle)

Let $a:=\operatorname{essinf}_{x \in \Omega} f(x)$ and $b:=\operatorname{ess}_{\sup _{x \in \Omega}} f(x)$. Then, $a \leq u(x, t) \leq b$ on $\Omega \times[0, \infty)$.

(c) (Average grey level invariance)

The average grey level $\mu:=\frac{1}{\Omega} \int_{\Omega} f(x) d x$ is not affected by nonlinear diffusion filtering: $\frac{1}{|\Omega|} \int_{\Omega} u(x, t) d x=\mu$ for all $t>0$.

(d) (Lyapunov functionals)

$V(t):=\Phi(u(t)):=\int_{\Omega} r(u(x, t)) d x$ is a Lyapunov function for all $r \in \mathrm{C}^{2}[a, b]$ with $r^{\prime \prime} \geq 0$ on $[a, b]: V(t)$ is decreasing and bounded from below by $\Phi(M f)$, where $(M f)(y):=\mu$ for all $y \in \Omega$.

(e) (Convergence to a constant steady state) $\lim _{t \rightarrow \infty}\|u(t)-M f\|_{L^{p}(\Omega)}=0$ for $1 \leq p<\infty$.

The well-posedness results in (a) have significant practical impact, as they guarantee the stability with respect to perturbations of the initial images. This is of importance when considering stereo images, image sequences or slices from medical CT or MRT sequences, since we know that similar images remain similar after filtering.

Many smoothing scale-space properties are closely related to extremum principles: Hummel [7] for instance shows that under certain conditions the maximum principle for parabolic operators is equivalent to the property that the corresponding scale-space never creates additional level-crossings for $t>0$.

Average grey level invariance is a property which distinguishes diffusion filters from morphological scale-spaces. In addition to this invariance it is evident that $\left(P_{d}\right)$ satisfies classical scale-space invariances like grey level shift invariance, reverse contrast invariance, translation invariance and isometry invariance (see [1] for precise definitions). Usual architectural properties of scale-spaces (e.g. the semi-group property) are satisfied as well.

The Lyapunov functionals introduced in (d) show that the considered evolution equation is a simplifying, information-reducing transform with respect to many aspects: Indeed, special choices for $r$ immediately imply that all $L^{p}$ norms with $2 \leq p \leq \infty$ are decreasing (e.g. the energy $\|u(t)\|_{\mathrm{L}^{2}(\Omega)}^{2}$ ), all even central moments are decreasing (e.g. the variance), and the entropy $S[u(t)]:=$ $-\int_{\Omega} u(x, t) \ln (u(x, t)) d x$ is increasing with respect to $t[16]$.

The result (e) tells us that, for $t \rightarrow \infty$, this simplifying scale-space representation tends to the most global image representation that is possible: a constant image with the same average grey level as $f$.

Interestingly, $\left(P_{c}\right)$ does not need any monotony assumption (comparison principle) [1]. This is in contrast to linear diffusion and morphological scale-spaces and allows nonlinear diffusion scale-spaces to reveal contrast-enhancing properties leading to segmentation-like results (see $[16,18]$ for examples). In this sense we 
have a scale-space framework which does not contradict certain image restoration demands.

\section{Semidiscrete Case}

Let us now establish conditions under which comparable well-posedness and scalespace results can be proved for the semidiscrete framework. This case is of special interest since it involves the spatial discretization which is characteristic for digital images but it keeps the scale-space idea of using a continuous scale parameter. It leads to nonlinear systems of ordinary differential equations.

A discrete image can be regarded as a vector $f \in \mathbb{R}^{N}, N \geq 2$, whose components $f_{j}, j=1, \ldots, N$ represent the grey values at the pixels. We denote the index set $\{1, \ldots, N\}$ by $J$. The semidiscrete problem class $\left(P_{s}\right)$ we are concerned with is defined as follows:

Let $f \in \mathbb{R}^{N}$. Find a function $u \in \mathrm{C}^{1}\left([0, \infty), \mathbb{R}^{N}\right)$ which satisfies the initial value problem

$$
\begin{aligned}
\frac{d u}{d t} & =A(u) u \\
u(0) & =f
\end{aligned}
$$

where $A=\left(a_{i j}\right)$ has the following properties:

(S1) Lipschitz-continuity of $A \in \mathrm{C}\left(\mathbb{R}^{N}, \mathbb{R}^{N \times N}\right)$ for every bounded subset of $\mathbb{R}^{N}$,

(S2) symmetry: $\quad a_{i j}(u)=a_{j i}(u) \quad \forall i, j \in J, \forall u \in \mathbb{R}^{N}$,

(S3) vanishing row sums: $\sum_{j \in J} a_{i j}(u)=0 \quad \forall i \in J, \forall u \in \mathbb{R}^{N}$,

(S4) nonnegative off-diagonals: $a_{i j}(u) \geq 0 \quad \forall i \neq j, \forall u \in \mathbb{R}^{N}$,

(S5) irreducibility for all $u \in \mathbb{R}^{N}$.

Under these prerequisites we obtain the subsequent well-posedness and scale-space results [18]:

Theorem 2 (Properties of the semidiscrete filter class)

For the semidiscrete filter class $\left(P_{s}\right)$ the following statements are valid:

(a) (Well-posedness)

For every $T>0$ the problem $\left(P_{s}\right)$ has a unique solution $u(t) \in \mathrm{C}^{1}\left([0, T], \mathbb{R}^{N}\right)$. This solution depends continuously on the initial value and the right-hand side of the $O D E$ system.

(b) (Extremum principle)

Let $a:=\min _{j \in J} f_{j}$ and $b:=\max _{j \in J} f_{j}$. Then, $a \leq u_{i}(t) \leq b$ for all $i \in J$ and $t \in[0, T]$.

(c) (Average grey level invariance)

The average grey level $\mu:=\frac{1}{N} \sum_{j \in J} f_{j}$ is not affected by the semidiscrete diffusion filter: $\frac{1}{N} \sum_{j \in J} u_{j}(t)=\mu$ for all $t>0$. 
(d) (Lyapunov functionals)

$V(t):=\Phi(u(t)):=\sum_{i \in J} r\left(u_{i}(t)\right)$ is a Lyapunov function for all $r \in \mathrm{C}^{1}[a, b]$ with increasing $r^{\prime}$ on $[a, b]: V(t)$ is decreasing and bounded from below by $\Phi(c)$, where $c:=(\mu, \ldots, \mu)^{\top} \in \mathbb{R}^{N}$.

(e) (Convergence to a constant steady state) $\lim _{t \rightarrow \infty} u(t)=c$.

These results allow the same interpretation as their continuous counterparts. Not all of the requirements (S1)-(S5) are necessary for each of the theoretical results above. (S1) is needed for well-posedness, the proof of a maximum-minimum principle involves (S3) and (S4), while average grey value invariance uses (S2) and (S3). The existence of Lyapunov functionals can be established by means of (S2)-(S4), and convergence to a constant steady state requires (S5) in addition to (S2)-(S4).

It can be shown [18] that there exist finite difference approximations of $\left(P_{c}\right)$ fulfilling the requirements (S1)-(S5) of $\left(P_{s}\right)$. However, $\left(P_{c}\right)$ is not the only family which leads to semidiscrete filters satisfying (S1)-(S5): Interestingly, a semidiscrete version of the Perona-Malik filter [13] (which is claimed to be ill-posed in the continuous setting [5]) on a fixed grid also satisfies (S1)-(S5) and, thus, reveals all the beforementioned well-posedness and scale-space properties. This is due to the fact that the extremum principle limits the modulus of discrete gradient approximations. Hence, the spatial discretization implicitly causes a regularization.

\section{Discrete Case}

In practice, scale-spaces are always approximated with a finite number of scales. This corresponds to the fully discrete case which shall be treated now. In this setting we impose the subsequent requirements:

Let $f \in \mathbb{R}^{N}$. Calculate a sequence $\left(u^{(k)}\right)_{k \in \mathbb{N}_{0}}$ of processed versions of $f$ by means of

$$
\begin{aligned}
u^{(0)} & =f, \\
u^{(k+1)} & =Q\left(u^{(k)}\right) u^{(k)}, \quad \forall k \in \mathbb{N}_{0},
\end{aligned}
$$

where $Q=\left(q_{i j}\right)$ has the following properties:

(D1) continuity in its argument:

$$
Q \in \mathrm{C}\left(\mathbb{R}^{N}, \mathbb{R}^{N \times N}\right),
$$

(D2) symmetry:

(D3) unit row sum: $q_{i j}(v)=q_{j i}(v) \quad \forall i, j \in J, \forall v \in \mathbb{R}^{N}$,

(D4) nonnegativity:

(D5) irreducibility for all $v \in \mathbb{R}^{N}$,

(D6) positive diagonal: $\sum_{j \in J} q_{i j}(v)=1 \quad \forall i \in J, \forall v \in \mathbb{R}^{N}$, $q_{i j}(v) \geq 0 \quad \forall i, j \in J, \forall v \in \mathbb{R}^{N}$, $\left.q_{i i}(v)>0 \quad \forall i \in J, \forall v \in \mathbb{R}^{N}.\right)$

This gives similar results as in the continuous and semidiscrete case [18]: 


\section{Theorem 3 (Properties of the discrete filter class)}

For the discrete filter class $\left(P_{d}\right)$ the following statements are valid:

(a) (Continuous dependence on initial image)

For every $k>0$ the unique solution $u^{(k)}$ of $\left(P_{d}\right)$ depends continuously on the initial image $f$.

(b) (Extremum principle)

Let $a:=\min _{j \in J} f_{j}$ and $b:=\max _{j \in J} f_{j}$. Then, $a \leq u_{i}^{(k)} \leq b$ for all $i \in J$ and $k \in \mathbb{N}_{0}$.

(c) (Average grey level invariance)

The average grey level $\mu:=\frac{1}{N} \sum_{j \in J} f_{j}$ is not affected by the discrete diffusion filter: $\frac{1}{N} \sum_{j \in J} u_{j}^{(k)}=\mu$ for all $k \in \mathbb{N}_{0}$.

(d) (Lyapunov functionals)

$V^{(k)}:=\Phi\left(u^{(k)}\right):=\sum_{i \in J} r\left(u_{i}^{(k)}\right)$ is a Lyapunov sequence for all convex $r \in \mathrm{C}[a, b]: V^{(k)}$ is decreasing and bounded from below by $\Phi(c)$, where $c:=(\mu, \ldots, \mu)^{\top} \in \mathbb{R}^{N}$.

(e) (Convergence to a constant steady state) $\lim _{k \rightarrow \infty} u^{(k)}=c$.

It can be shown [18] that the semi-implicit scheme

$$
\frac{u^{(k+1)}-u^{(k)}}{\tau}=A\left(u^{(k)}\right) u^{(k+1)}
$$

with $A$ satisfying (S1)-(S5) fulfils the prerequisites (D1)-(D6) for discrete diffusion models for every positive time step size $\tau$. Hence, $\left(P_{d}\right)$ arises in a natural way from $\left(P_{s}\right){ }^{3}$ On the other hand, the assumptions (S1)-(S5) are sufficient conditions for the semi-implicit scheme to fulfil (D1)-(D6), but they are not necessary: Nonnegativity of $Q\left(u^{(k)}\right)$ may also be achieved using spatial discretizations where $A\left(u^{(k)}\right)$ violates nonnegativity (see [4] for examples).

\section{$5 \quad$ Summary and Conclusions}

We have investigated a complete scale-space framework for nonlinear diffusion filtering in the continuous, semidiscrete and discrete setting. This is of special importance, since besides a few exceptions $[10,6]$ the practically relevant problem of how to design (semi-)discrete scale-spaces has hardly been addressed in the literature.

We have established conditions under which one can prove well-posedness and scale-space results for nonlinear diffusion filtering. In all three settings we have used similar assumptions: smoothness ((C1), (S1), (D1)), symmetry ((C2), (S2),

\footnotetext{
${ }^{3}$ Explicit and $\alpha$-semi-implicit schemes satisfy (D1)-(D6) as well, provided that some time step size restrictions are imposed (see [18] for more details).
} 
(D2)), nonnegativity ((C4), (S4), (D4)), requirements ensuring a nonvanishing diffusion at all locations ((C3), (S5), (D5)-(D6)) and assumptions expressing the conservation of the average grey level (divergence form and boundary condition in the continuous case, (S3), (D3)). However, it should be observed that the smoothness assumptions can be weakened during the transition from the coontinuous to the discrete framework. This is also true for the convex function $r$ which generates the smoothing Lyapunov functionals.

\section{References}

[1] Alvarez, L., Guichard, F., Lions, P.-L., Morel, J.-M.: Axioms and fundamental equations in image processing. Arch. Rat. Mech. Anal. 123 (1993) 199-257

[2] Ballester Nicolau, C.: An affine invariant model for image segmentation: Mathematical analysis and applications. Ph.D. thesis, University of Illes Baleares, Palma de Mallorca, Spain, 1995

[3] Bigün, J., Granlund, G.H., Wiklund, J.: Multidimensional orientation estimation with applications to texture analysis and optical flow. IEEE Trans. Pattern Anal. Mach. Intell. 13 (1991) 775-790

[4] Bramble, J.H., Hubbard, B.E.: New monotone type approximations for elliptic problems. Math. Comp. 18 (1964) 349-367

[5] Catté, F., Lions, P.-L., Morel, J.-M., Coll, T.: Image selective smoothing and edge detection by nonlinear diffusion. SIAM J. Numer. Anal. 29 (1992) $182-193$

[6] Cohignac, T., Eve, F., Guichard, F., Lopez, C. Morel, J.-M.: Numerical analysis of the fundamental equation of image processing. Preprint No. 9254, CEREMADE, Université Paris IX - Dauphine, Place du Maréchal de Lattre de Tassigny, 75775 Paris Cedex 16, France, 1992

[7] Hummel, R.A.: Representations based on zero-crossings in scale space. Proc. IEEE Comp. Soc. Conf. Computer Vision and Pattern Recognition (CVPR '86, Miami Beach, June 22-26, 1986), IEEE Computer Society Press, Washington, 204-209, 1986

[8] Jähne, B.: Spatio-temporal image processing. Lecture Notes in Comp. Science, Vol. 751, Springer, Berlin, 1993

[9] Koenderink, J.J.: The structure of images. Biol. Cybern. 50 (1984) 363-370

[10] Lindeberg, T.: Scale-space for discrete signals. IEEE Trans. Pattern Anal. Mach. Intell. 12 (1990) 234-254

[11] Lindeberg, T.: Scale-space theory in computer vision. Kluwer, Boston, 1994 
[12] Nitzberg, M., Shiota, T.: Nonlinear image filtering with edge and corner enhancement. IEEE Trans. Pattern Anal. Mach. Intell. 14 (1992) 826-833

[13] Perona, P., Malik, J.: Scale space and edge detection using anisotropic diffusion. IEEE Trans. Pattern Anal. Mach. Intell. 12 (1990) 629-639

[14] Rao, A.R., Schunck, B.G.: Computing oriented texture fields. CVGIP: Graphical Models and Image Processing, 53 (1991) 157-185

[15] Weickert, J.: Anisotropic diffusion filters for image processing based quality control. In Fasano, A., Primicerio, M. (Eds.): Proc. Seventh European Conf. on Mathematics in Industry. Teubner, Stuttgart, 355-362, 1994

[16] Weickert, J.: Scale-space properties of nonlinear diffusion filtering with a diffusion tensor. Report No. 110. Laboratory of Technomathematics, University of Kaiserslautern, P.O. Box 3049, 67653 Kaiserslautern, Germany, 1994 (submitted)

[17] Weickert, J.: Multiscale texture enhancement. V. Hlaváč, R. Šára (Eds.), Computer analysis of images and patterns, Lecture Notes in Comp. Science, Vol. 970, Springer, Berlin, 230-237, 1995

[18] Weickert, J.: Anisotropic diffusion in image processing. Ph.D. thesis, Dept. of Mathematics, University of Kaiserslautern, Germany, January 1996

[19] Whitaker, R.T., Pizer, S.M.: A multi-scale approach to nonuniform diffusion. CVGIP: Image Understanding 57 (1993) 99-110 